\title{
Extracting Structure Information from TDHF
}

\author{
P. D. Stevenson and S. Fracasso \\ Dept. of Physics, University of Surrey, Guildford, Surrey, GU2 7XH, United Kingdom \\ E-mail: p.stevenson@surrey.ac.uk
}

\begin{abstract}
This contribution to the "Open Problems in Nuclear Structure Theory" special edition looks at some issues with using Time-Dependent Hartree Fock and related techniques to study structural phenomena in nuclear physics. We limit the discussion to structures like giant resonances and discuss some open questions regarding the interpretation of TDHF calculations.
\end{abstract}

\section{Introduction}

The time-dependent Hartree-Fock (TDHF) approach was written down by Dirac in the 1930s [1] but first applied in anger to nuclear physics problems in the 1970s. As a method able to deal with large amplitude motion, the application of TDHF has been most prominent in reaction dynamics. Though such studies shed much light on nuclear structure as well as reaction mechanisms, we concentrate in this contribution on the use of TDHF in studies of giant resonances in which one is purely concerned with the structure of a single nuclide.

Giant resonances are collective excitation modes, occurring largely above the particle emission threshold and usually characterised by their multipolarity and spin and isospin character. The basic microscopic approach (within the realm of mean field models) to calculating giant resonances is the Random Phase Approximation (RPA). The RPA can be derived as the small-amplitude limit of TDHF, so in principle TDHF should do all RPA does and more. Usually RPA calculations of giant resonances calculate the strength function defined as

$$
S(E)=\sum_{\nu}|\langle\nu|\hat{F}| 0\rangle|^{2} \delta\left(E-E_{\nu}\right)
$$

where $\hat{F}$ is the operator describing the excitation mode of interest. In TDHF, this quantity is calculated by exciting the system with the operator $\hat{F}$ and following the time-dependent expectation value of the same operator $\langle\hat{F}\rangle(t)$. According to linear response theory, the strength function can be calculated in TDHF as [2]

$$
S(E)=-\frac{1}{\pi} \operatorname{Im}\left(\frac{\tilde{F}(E)}{\tilde{f}(E)}\right),
$$


where $\tilde{F}(E)=\tilde{F}(\hbar \omega)$ is the Fourier transform of the time-dependent response $\langle\hat{F}\rangle(t)$ and $\tilde{f}(E)=\tilde{f}(\hbar \omega)$ is the Fourier transform of the time-dependence of the external excitation $\hat{F}$ causing the resonance. Note that the expression (2) holds under the assumption that the response depends linearly on the strength of the external field $[3,4]$.

Early TDHF calculations for giant resonances concentrated on monopole resonances $[5,6,7]$ since they require only a spherical one-dimensional code, and were also restricted to simplified forms of effective interactions. However, the principles of extracting information from the calculations remain the same as more powerful computers led to the ability to calculate giant resonances with non-spherical instantaneous deformations and those built on nuclei of arbitrary shape $[8,9,10,11,12]$. In addition to the increase in computer power allowing the relaxation of symmetry assumptions, it is also the combined sophistication and simplicity of the Skyrme-type interactions that allow useful TDHF calculations to take place [13].

\section{Observables, Excitation Operators and Modes}

A general question that arises in the study of giant resonances is what are the interesting quantities to calculate? As mentioned, the standard quantity calculated in RPA is the strength function, which can be related to an experimental cross section, though it is not an instrinsic property of the nucleus, but also on the particular experiment and the associated momentum transfer $[7,14]$. One interesting possibility that TDHF allows is the direct simulation of a realistic time-profile for the excitation and an analysis of the results, or even the direct simulation of a collision. It is an open question whether one will get results more closely related to the experimental situation, but there is scope to try.

A typical RPA analysis of the strength looks also at individual contributions from particular particles in the basis of the uncorrelated ground state. This is less straightforward to do in TDHF. In practical TDHF calculations a large basis of unperturbed single particles states is not needed (or desirable) as the excitations are realised by time-evolved single particle states with a different Slater Determinant at each time. Combining this set of Slater Determinants by means of a Fourier transform then corresponds to the RPA case. By an analysis of the excitation energies, though, it can be possible to identify the dominant single particle state in a structure in the strength function [15], when indeed a single one is dominant. There is no analogue in TDHF with the so-called unperturbed RPA response. However, one can be imaginative in the way that one excites the nucleus. Figure 1 shows the giant monopole resonance in ${ }^{16} \mathrm{O}$ using the $\mathrm{SkM}^{*}$ force in which the total response is shown, alongside the response of the $\pi 1 \mathrm{~s}$ orbital to an excitation of only the $\pi 1$ s orbital, which shows that the $\pi 1 \mathrm{~s}$ orbital is responsible for the structure around $30 \mathrm{MeV}$. The interpretation, however, is not trival. The excitation of the $\pi 1 \mathrm{~s}$ state induces oscillations in the other single particle states. This overall excitation is different to the total GMR excitation, so the peak positions do not line up exactly. Unlike in RPA, we cannot easily disentangle the contributions 


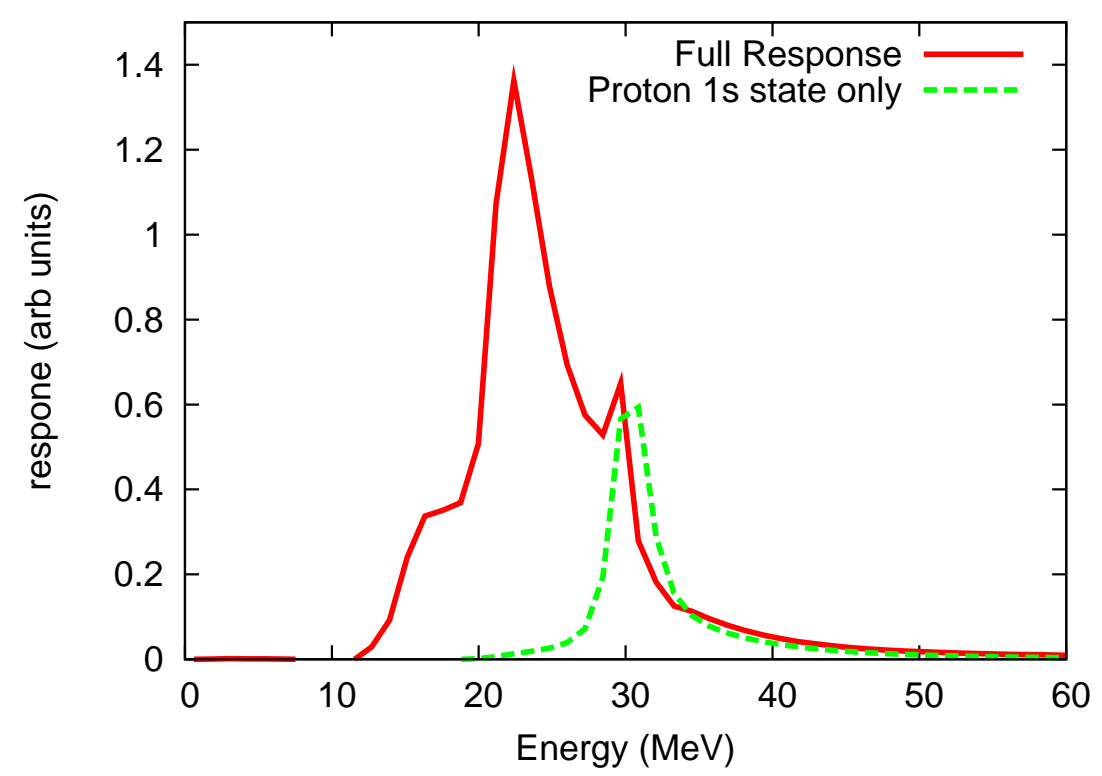

Figure 1. The total giant monopole response ("Full Response") in $16 \mathrm{O}$ and the response of the proton 1s state to that state alone being excited. The calculation is a continuum calculation since the edge of the box is not reached by the flux up until the final time of $1024 \mathrm{fm} / \mathrm{c}$. This relatively small final time gives an energy resolution of $\sim 1.2 \mathrm{MeV}$.

from each particle state. Comparing with RPA calculations can therefore be difficult. In RPA, moreover, one can isolate the contribution to the response easily from different parts of the residual interaction, which, as well-known is obtained as a second derivative of the Hartree-Fock energy density expressd in terms of the static densities. In TDHF one does not explicitly construt the residual interaction, the only ingredient being the time-dependent mean-field, and the ability to understand which parts of the interaction are contributing is understood in a different way. One cannot simply count up which parts of the underlying interaction in TDHF give rise to the strength as in RPA.

As well as a straightforward multipolar decomposition, there are many possibilities for combining space and time functions of the excitation of the nucleus that are allowed by TDHF - e.g. one can excite only the surface, only protons, only specific orbitals, with angular momentum, with a sudden kick, with a fast or slow external field or a harmonic driving force, all of which can give information about the structure of the nucleus. One can also look for new kinds of excitation that may arise such as the axial spin-twist mode [16], though how to think of a clean signal for its observation is another matter.

TDHF with no spatial constraints permits, and demands, a completely general spatial response to a particular excitation. One cannot (in general) give a nucleus a monopole excitation and expect no quadrupole to be excited in addition. At any given frequency, one might attempt to find the normal modes of excitation, presumably in a multipolarity basis [17]. An example of this mode mixing is easily seen in monopole calculations of the inducement of isovector modes to isoscalar excitations and vice versa [18]. 
The study of giant resonances and collective modes (by whatever theoretical method) has been of considerable use in understanding the link between effective interactions and the properties of nuclear matter [4] and has been used for understanding how effective interactions fitted to ground state data can be further constrained by excited state properties [19, 20, 21]. What further role can TDHF play in this area? Constraining those terms in the functional which are made of time-odd densities, including those that arise only from the tensor force [22], is certainly now a possibility given the existing TDHF codes, and a suitable suite of observables needs to be chosen. Nuclear matter properties can also be probed in collisions and TDHF gives an ideal method to combine understanding the properties of nuclear matter simultaneously on structure and reaction observables.

As a final point about the comparison between RPA and TDHF, we bring up the ground state energy correlations. As is well known, particle-hole correlations are explicitly taken into account in RPA, contributing through the $Y$ amplitudes to the response function and the ground state energy [24]. To the best of our knowledge, no attempt has been made to realise an approach to use TDHF to calculte the same correlated ground state as in RPA [25], though some interesting uses are made of time-dependent methods to build in certain classes of ground state correlations [26]. Today's symmetry unrestricted TDHF codes could in principle be used to build in more sophisticated correlations.

\section{Nonlinearities}

TDHF is inherently able to deal with nonlinearities in the sense that the nuclear response to an excitation is not, in TDHF, necessarily proportional to the magnitude of the excitation. As an example of the kind of size of effect that appears, the nonlinear response of a monpole excitation in ${ }^{16} \mathrm{O}$ is presented in figure 2. In extracting information from the nonlinear regime, different groups have taken different approaches, including a spectral analysis [15], an exploration of the coupling matrix elements between different modes [17], an information-theoretic approach [27], in terms of quasiperiodic orbits [28], or the analysis of level-spacing [18]. There has, so far, been little attempt to unify or compare different approaches to exploring nonlinearities - though the informationtheoretic approaches are looking more at the inherent nonlinearities of the TDHF equations than the linearity of the response. As well as a lack of comparison of existing methods for exploring nonlinearities, we are not aware of work coupling particlevibration coupling with time-depdendence.

\section{The Continuum and the Open Quantum System}

The two topics of the continuum and open quantum systems are related in that giant resonances can decay by particle emission. TDHF inherently preserves the norm of the wavefunction and so to study particle emission in TDHF one must couple the nucleus 
to the environment and allow emitted particles to disappear into the continuum. The easiest way of doing this is to use a large coordinate space grid in which the normal TDHF equations are solved, but consider a small region of the grid in which the nucleus is said to exist - and in which region alone nuclear observables are calculated [7]. This is practical only in one-dimension [7, 9]. In a full three-dimensional calculation such large boxes become prohibitive. Non-uniform gridding and absorbing boundaries can help [12], though it cannot give an exact solution. One possible route to a proper treatment of the continuum is to use some form of exact boundary condition [29]. It is an open question whether such an approach will be sufficiently efficient in the case of 3D TDHF.

Note that it is the reflecting boundaries and the discretisation of the continuum that allows for the kind of nonlinear analysis in [18] and [27]. It also allows for the construction of periodic orbits. If the system is treated as an open quantum systems with nucleus and environment, then dealing with periodic orbits for the nucleus only will require some serious thought.

The process of separating the space into a nucleus and the environment is necessary to treat the continuum. It has its problems, though, in that the point at which to make the cut is chosen with rules of thumb, and results depend slightly on where the choice is made. As an example, Figure 3 shows the time-evolution of the isoscalar giant monopole moment for ${ }^{16} \mathrm{O}$ with a kick size of $0.0001 \mathrm{fm}^{-2}$, in a $240 \mathrm{fm}$ box with time evolved to $1024 \mathrm{fm} / \mathrm{c}$ (and no flux hitting the edge of the box). The different lines show the result of making different choices for the cut point. In detail, no two lines quite agree with each other. The resulting Fourier transforms are shown, and show that the effect on

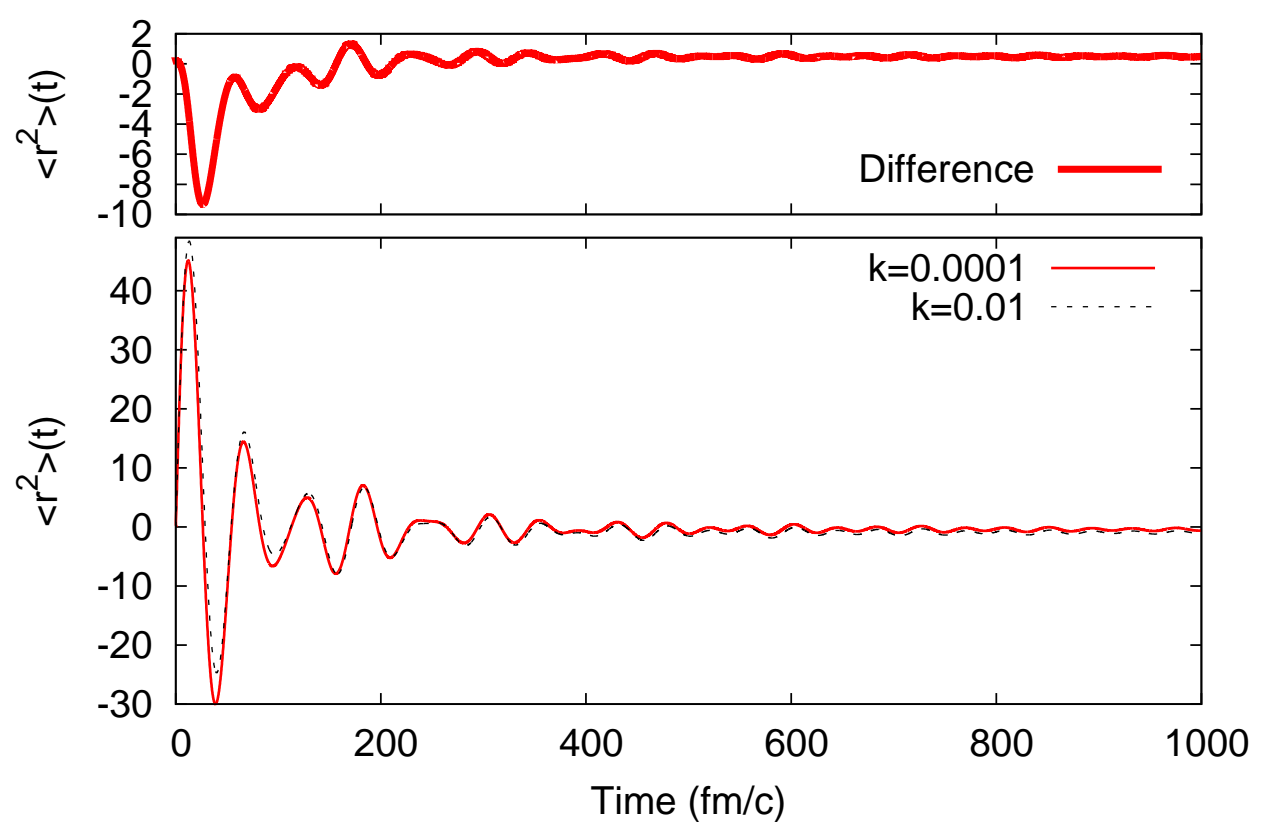

Figure 2. Nonlinearities induced by a strong instantaneous boost with kick size $k=0.01 \mathrm{fm}^{-2}$ compared with a smaller kick in the linear regime 

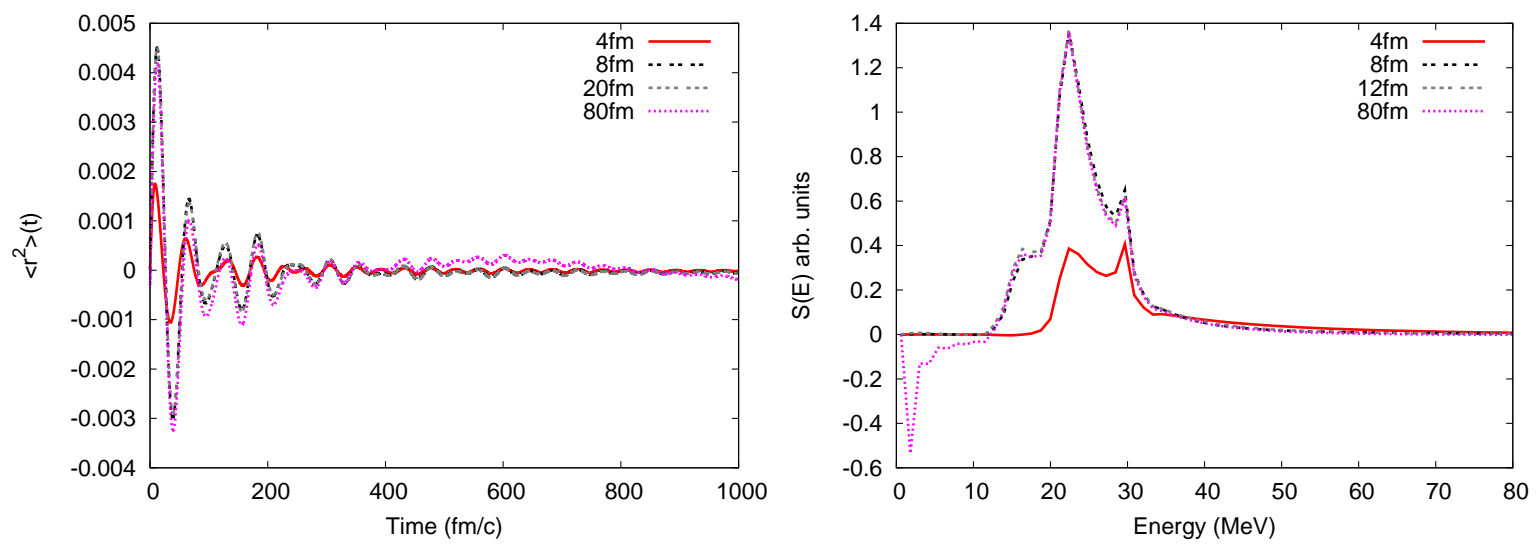

Figure 3. The response of ${ }^{16} \mathrm{O}$ to an isoscalar monopole excitation, with the meansquare radius measured out to a finite cut-off which is less than the total size of the box. The left plot show the time-dependence of the mean-square radius, and the right plot the Fourier transform.

the strength is not strong, providing the cutoff between nucleus and environment is taken at a large enough radius. Larger radii make nonlinearities more obvious for the same external perturbation size, as indicated by the failure of the Fourier transform to produce a positive function, though the strength in the peak region is remarkably unchanged.

A better understanding of how TDHF can be interpreted as describing an open quantum system is now called for, alongside a comparison to methods which explicitly add an environmental coupling [30].

\section{Beyond TDHF}

In a sense, we have already talked about going "beyond TDHF" in considering the entire nucleus + environment system as obeying the TDHF equations, but analysing a restricted part of the density matrix and identfying it with the nucleus, thus allowing the study of particle emission within a framework that preserves norm. However, there are many ways one would like to go more genuinely beyond TDHF to improve our understanding of nuclear structure. It is well-known that TDHF misses the collisional width in giant resonances associated with $2 \mathrm{p}-2 \mathrm{~h}$ (and higher) collisions. Various methods exist, which have TDHF as a base, which can be used to look beyond TDHF [31, 32, 33], including the inclusion of pairing, which is naturally an important ingredient for structure studies [34]. The time is now ripe for some serious comparisons based on the current generation of sophisticated codes.

\section{Conclusion}

We have outlined a few areas in which questions exist in the use of TDHF for nuclear structure calculations. Many of these relate to the comparison of TDHF to 
other methods. Since the recent advent of symmetry-unrestricted TDHF codes, these questions will be able to be tackled.

\section{Acknowledgements}

This work was supported by the UK Science and Technology Facilities Council under grant number PP/F000596/1.

\section{References}

[1] P. A. M. Dirac, Proc. Camb. Philos. Soc. 26, 376 (1930)

[2] P.-G. Reinhard, P. D. Stevenson, D. Almehed, J. A. Maruhn and M. R. Strayer, Phys. Rev. E 73, 036709 (2006)

[3] A. L. Fetter and J. D. Walecka, Quantum Theory of Many-Particle Systems, McGraw Hill, St Louis (1971).

[4] J. P. Blaizot, Phys. Rep. 64, 171 (1980)

[5] J. Błocki and H. Flocard, Phys. Lett. B 85, 163 (1979)

[6] S. Stringari and D. Vautherin, Phys. Lett. B 88, 1 (1979)

[7] Ph. Chomaz, Nguyen Van Giai and S. Stringari, Phys. Lett. B 189, 375 (1987)

[8] C. R. Chinn, A. S. Umar, M. Valliéres and M. R. Strayer, Phys. Rep. 264, 107 (1996)

[9] P. D. Stevenson, M. R. Strayer, J. Rikovska Stone and W. G. Newton, Int. J. Mod. Phys. E 13, $181(2004)$

[10] M. P. Brine, P. D. Stevenson, J. A. Maruhn and P.-G. Reinhard, Int. J. Mod. Phys. E 15, 1417 (2006)

[11] J. A. Maruhn, P.-G. Reinhard, P. D. Stevenson, J. Rikovska Stone and M. R. Strayer, Phys. Rev. C 71, 064328 (2005)

[12] Takashi Nakatsukasa and Kazuhiro Yabana, Phys. Rev. C 71, 024301 (2005)

[13] Y. M. Engel, D. M. Brink, K. Goeke, S. J. Krieger and D. Vautherin, Nucl. Phys. A 249 (1975)

[14] J. Dechargé, D. Gogny, B. Grammaticos and L. Šips, Phys. Rev. Lett. 49, 982 (1982)

[15] P.-G. Reinhard, Lu Guo and J. A. Maruhn, Eur. Phys. J.A 32, 19 (2007)

[16] J. A. Maruhn, P.-G. Reinhard, P. D. Stevenson and M. R. Strayer, Phys. Rev. C 74, 027601 (2006)

[17] C. Simenel and Ph. Chomaz, Phys. Rev. C 80, 064309 (2009)

[18] P. D. Stevenson, D. Almehed, P.-G. Reinhard and J. A. Maruhn, Nucl. Phys. A 788, 343c (2007)

[19] S. Fracasso and G. Colò, Phys. Rev. C 76, 044307 (2007)

[20] Jun Li, Gianluca Colò and Jie Meng, Phys. Rev. C 78, 064304 (2008)

[21] Luca Trippa, Gianluca Colò and Enrico Vigezzi, Phys. Rev. C 77, 061304(R) (2008)

[22] E. Perlińska, S. G. Rohoziński, J. Dobaczewski and W. Nazarewicz, Phys. Rev. C 69, 014316 (2004)

[23] F. Dönau, D. Almehed and R. G. Nazmitdinov, Phys. Rev. Lett. 83, 280 (1999)

[24] D. J. Rowe, Phys. Rev. 175, 1283 (1968)

[25] S. Baroni, M. Armati, F. Barranco, R. A. Brogia, G.Colò, G. Gori and E. Vigezzi, J. Phys. G 30, $1353(2004)$

[26] Marlène Assié and Denis Lacroix, Phys. Rev. Lett. 102, 202501 (2009)

[27] G. A. Lalazissis, D. Vretenar, N. Paar and P. Ring, Chaos, Solitons and Fractals 17, 585 (2003)

[28] A. S. Umar, M. R. Strayer, R. Y. Cusson, P.-G. Reinhard and D. A. Bromley, Phys. Rev. C 32, $172(1985)$

[29] M. Mangin-Brinet, J. Carbonell, and C. Gignoux, Phys. Rev. A57, 3245 (1998)

[30] A. Diaz-Torres, D. J. Hinde, M. Dasgupta, G. J. Milburn and J. A. Tostevin, Phys. Rev. C 78, $064604(2008)$

[31] P.-G. Reinhard, R. Y. Cusson and K. Goeke, Nucl. Phys. A 398, 141 (1983) 
[32] J. M. A. Broomfield and P. D. Stevenson, J. Phys. G 35, 095102 (2008)

[33] Sakir Ayik, Kouhei Washiyama and Denis Lacroix, Phys. Rev. C 79, 054606 (2009)

[34] B. Avez, C. Simenel and Ph. Chomaz, Phys. Rev. C 78, 044318 (2009) 